\title{
A Physical Limit to the Magnetic Fields of T Tauri Stars
}

\author{
Pedro N. Safier \\ University of Maryland, \\ Laboratory for Millimeter-Wave Astronomy, \\ College Park, MD 20742; \\ e-mail: safier@astro.umd.edu
}

\begin{abstract}
Recent estimates of magnetic field strengths in T Tauri stars yield values $B=1-4 \mathrm{kG}$. In this paper, I present an upper limit to the photospheric values of $B$ by computing the equipartition values for different surface gravities and effective temperatures. The values of $B$ derived from the observations exceed this limit, and I examine the possible causes for this discrepancy.
\end{abstract}

Subject headings: stars: atmospheres - stars: late-type - stars: magnetic fields - stars: pre-main sequence

To appear in The Astrophysical Journal Letters 


\section{Introduction}

Magnetic fields are believed to play a fundamental role in the structure and evolution of $\mathrm{T}$ Tauri stars. Unfortunately, their detection and measurement in these objects is a very difficult task.

Early attempts to detect magnetic fields in T Tauri stars through Zeeman polarization were unsuccessful (Johnstone \& Penston 1986, 1987), and only recently such detections are forthcoming through the measurement of Zeeman broadening of photospheric lines in a handful of objects ( Basri et al. 1992; Guenther 1997). These measurements yield disk-averaged, photospheric field strengths of order a few $\mathrm{kG}$.

These observations do not provide a direct measurement of $B$; instead, what is measured is the difference in broadening of magnetically-sensitive and insensitive lines (Robinson 1980; Marcy 1982; Saar 1988). To find the value of $B$, one has to fit the data with a stellar atmosphere model and vary $B$ and $f$ - the fraction of stellar surface that is magnetizeduntil a good match is found. The result of this matching procedure is the product $f B$, and independent determination of $B$ and $f$ is much more problematic and sensitive to modeling assumptions (see, e.g., Solanki 1992 and references therein). Furthermore, when equivalent widths are used in lieu of measuring the line broadening, the deduced $f B$ is also very sensitive to the input model atmosphere (Basri et al. 1992; Basri \& Marcy 1994).

The purpose of this paper is to present a physical constraint on the maximum value of $f B$; this upper limit on the magnetic field strength is important to estimate $B$ from the $f B$-values obtained from the data, and may help to uncover potential errors in the modeling assumptions.

The basic physics is rather well known, and has been successfuly applied to the study of the Sun. Magnetic fields are excluded from closed circulation patterns when the field lines are perpendicular to the axis of rotation of the fluid (see, e.g., Parker 1979, ch. 16 and references therein); as a result of this general principle the solar convection sweeps the field into the downdraft regions of the eddies. Convective heat transport is strongly inhibited within the field (Biermann 1941), and, through the so-called superadiabatic effect (Parker 1978), the downflowing gas further cools and evacuates the magnetized gas; as a result, the external gas pressure is larger than that inside the tube, and the flux tube shrinks until the to- tal internal pressure (thermal plus magnetic) balances the external pressure. Thus, the solar magnetic field is broken up and compressed into highly evacuated flux tubes.

Although we cannot resolve features on the surface of other stars, it is a reasonable inference that, in complete analogy with the Sun, the magnetic field in stars with outer convection zones - or convective throughout, as in the case of T Tauri stars - is concentrated into discrete flux tubes, which are in pressure equilibrium with the unmagnetized gas. If this is true, then an upper limit on $B$ can be derived if the pressure of the unmagnetized gas is known and the gas pressure inside the flux tubes is ignored ${ }^{1}$. These arguments have been extensively applied to active dwarfs (see, e.g., Saar 1996), but, so far, they have never been used to study the magnetic fields in T Tauri stars.

In $\S 2$ I derive an expression for the maximum equipartition value of $B$ as a function of the stellar and atmospheric parameters, and in $\S 3$ I compare the results from $\S 2$ with current observational estimates. My conclusions follow in $\S 4$.

\section{Equipartition Fields}

Consider a straight flux tube that is initially perpendicular to the photospheric surface at some fiducial height. The condition of lateral pressure equilibrium, i.e., the flux tube neither expands nor contracts, is given by

$$
\frac{B^{2}}{8 \pi}=\Delta P
$$

where $\Delta P$ is the difference between the external and internal non-magnetic pressures, $P_{i}$ and $P_{e}$, respectively, and the external medium is unmagnetized. At any height, an upper limit to $B$ is given by the equipartition field $\propto\left(P_{e}\right)^{1 / 2}$, and because temperature and pressure decrease with height in the photosphere, the maximum detectable field is the equipar-

\footnotetext{
${ }^{1}$ Note that this argument does not apply to stars with outer radiative zones, where there are no known mechanisms to isolate and concentrate the field. The Ap stars are a prime example, with measured fields as high as $30 \mathrm{kG}$ in the case of HD 215441 (Babcock 1960), a value much larger than that obtained by assuming pressure balance. Therefore, these fields are likely to thread the entire surface, and probably are fossil, rather than dynamo-generated, fields. In the case of $\mathrm{T}$ Tauri stars, after the onset of convection the fossil field is quickly destroyed by the resulting turbulent difussivity. Küker \& Rüdiger (1997) estimate that in a typical TTS the fossil field is destroyed in $\sim 300 \mathrm{yr}$ after the onset of convection. Therefore, the magnetic field in TTS must be dynamo-generated.
} 
tition field at the optical depth where the continuum is formed $(\tau=2 / 3)$,

$$
B_{\mathrm{eq}}=\left[8 \pi P_{e}(\tau=2 / 3)\right]^{1 / 2} .
$$

In principle, $P_{e}$ includes the contributions from both thermal pressure and ram pressure due to convective motions $\left(P_{T}\right.$ and $P_{v}$, respectively) because in a convective atmosphere the magnetic field outside dark spots is concentrated where the convective downdrafts are located (see, e.g., Stein et al., 1992). However, it is easy to show that $P_{T} \gg P_{v}$, and the argument is the following.

The ratio $P_{v} / P_{T}$ can be written as

$$
\frac{P_{v}}{P_{T}}=\frac{1}{3} \gamma\left(\frac{\bar{v}}{v_{s}}\right)^{2}
$$

where $\gamma$ is the ratio of the specific heat capacities, $\bar{v}$ is the average speed of a (turbulent) convective element, and $v_{s}$ is the adiabatic speed of sound. Although recent simulations of compressible convection (Cattaneo \& Malagoli, 1992) show that horizontal surface flows can be intermittently transonic, the downflows are subsonic, and, because $\gamma \leq 5 / 3$, it follows that $P_{v} / P_{T} \leq 5 / 9$. A more stringent limit on this ratio is given by estimating $\bar{v} / v_{s}$ using the mixing-length theory of convection, which gives (see, e.g., Cox \& Giuli 1968, eq. 14.64)

$$
\frac{\bar{v}}{v_{s}}=0.4\left(\frac{L_{*}}{1.4 \mathrm{~L}_{\odot}}\right)^{1 / 3}\left(\frac{T_{\mathrm{eff}}}{4000 \mathrm{~K}}\right)^{-1 / 2}\left(\frac{\mu}{1.7}\right)^{1 / 2}
$$

where $L_{*}, T_{\text {eff }}$, and $\mu$ are, respectively, the stellar luminosity, effective temperature, and photospheric mean molecular weight. Therefore, for the stellar parameters of a typical TTS, $P_{v} / P_{T} \approx 0.2$, and by neglecting $P_{v}$ the error introduced in $B_{\text {eq }}$ is $\sim 10 \%$ much smaller than the observational errors (see below). Henceforth I will neglect the contribution of ram pressure ${ }^{2}$ to $B_{\text {eq }}$, and use $P_{e}=P_{T}$.

The value of $P_{e}(\tau=2 / 3)$ follows from the condition of hydrostatic equilibrium, and to a very good degree of approximation (generally, better than a factor of 2 or 3; see, e.g., Cox \& Giuli 1968), one can write

$$
P_{e}(\tau=2 / 3)=\frac{2}{3} \frac{g}{\kappa}
$$

\footnotetext{
${ }^{2}$ The same result, essentialy, applies to the Sun, where the Maxwell stresses at the surface are an order of magnitude larger than the Reynolds stresses.
}

where $\kappa$ is the Rosseland mean opacity. The gas temperature $T$ at $\tau=2 / 3$ is assumed to be the effective temperature of the star, $T_{\text {eff }}$, and, because $\kappa$ is a function of density $\rho$ and $T$, eq. (5) is an implicit equation for $\rho\left(T=T_{\text {eff }}\right)$ for a given $T_{\text {eff }}, g$, and $\kappa\left(\rho, T_{\text {eff }}\right)$. Thus, one finally obtains

$$
B_{\mathrm{eq}}=\left[\frac{16 \pi}{3} \frac{g}{\kappa}\right]^{1 / 2} .
$$

I have computed $B_{\text {eq }}$ for different values of $T_{\text {eff }}$ and $g$ by solving eq. (5) using the Alexander \& Ferguson (1994) Rosseland mean opacities, and using eq. (6). To gauge the magnitudes of the errors introduced by the approximation used to derive eq. (5), I have also computed $B_{\text {eq }}$ by obtaining $P_{e}\left(T=T_{\text {eff }}\right)$ from the detailed stellar-atmosphere models by Guenther et. al (1992) and Allard \& Hauschildt (1995). The comparison between the two sets of $B_{\text {eq }}$ so obtained is presented in Table 1.

The results in Table 1 show that for spectral type $\mathrm{K} 5$, or earlier, the approximation used in eq. (5) results in an underestimate of the field by less than $10 \%$. On the other hand, for later spectral types the field is overestimated by, at most, a factor of 2 , but this error decreases with decreasing gravity. The reason for this larger discrepancy at cooler temperatures is the increase in opacity due to $\mathrm{H}_{2} \mathrm{O}$ once the gas temperature drops below $3500 \mathrm{~K}$ (Alexander \& Ferguson 1994), which results in a strong non-monotonic behavior of $\kappa$ with optical depth in the outer layers.

Therefore, for the typical gravities of $\mathrm{T}$ Tauri stars $(\log g \lesssim 4.0)$ and the spectral types currently accessible to Zeeman-broadening measurements (spectral type $\mathrm{K} 7$ or earlier), the equipartition fields predicted from eq. (6) are too small by, at most, $10 \%$ when compared with detailed atmospheric models.. These errors are much smaller than the error bars for the measured $f B$ 's (see Figure 1 below).

It is important to keep in mind that the measured field depends on the geometrical depth where the emission originates. Because flux tubes are strongly evacuated, the optical depth inside a magnetized region is smaller than in the surrounding atmosphere. If the flux tube is slender enough, it will be mostly transparent to the radiation crossing its walls, and, because of its lower density, it may appear as a bright feature against the continuum from its surroundings, as the network fields in the Sun do. In the solar case, the enhanced temperature compensates for the lower 
TABLE 1

Comparison Between $B_{\text {eq }}$ From Equation (6) and Detailed Stellar ATMOSPHERES

\begin{tabular}{lccrl}
\hline \hline Sp. Type & $\log g$ & $B_{\text {eq }}(\mathrm{kG})^{\mathrm{a}}$ & $\Delta^{\mathrm{b}}$ & \multicolumn{1}{c}{ Atmospheric Model } \\
\hline Sun & 4.4 & 1.2 & 0.08 & Guenther et al. 1992 \\
K5 & 4.5 & 1.8 & 0.06 & Allard \& Hauschildt 1995 \\
M1 & 4.0 & 1.5 & -0.13 & Allard \& Hauschildt 1995 \\
M5 & 4.0 & 1.5 & -0.36 & Allard \& Hauschildt 1995 \\
M5 & 5.0 & 4.8 & -0.52 & Allard \& Hauschildt 1995 \\
\hline
\end{tabular}

${ }^{\mathrm{a}}$ Computed from eq. (6).

${ }^{\mathrm{b}}$ Fractional difference; the equipartition field computed from a detailed atmospheric model is given by $B_{\text {eq }}[1+\Delta]$.

optical depth, and the values of $B$ measured in these bright points compare well with $B_{\text {eq }}\left(T=T_{\text {eff }}\right)$. If the Sun is any guidance, then $B_{\text {eq }}\left(T=T_{\text {eff }}\right)$ should be also a good approximation to the fields outside spots in other stars.

On the other hand, larger features like sunspots are much more evacuated and cooler than slender flux tubes, and at a fixed geometrical height the optical depth is much smaller inside the spot. Thus, one can see deeper into a sunspot, and they indeed appear as dimples on the Sun's surface when seen near the limb - a phenomenon known as the Wilson depression. Because the continuum from a spot originates at a larger geometrical depth, the external pressure there is larger, and, therefore, the fields measured inside sunspots are larger than those from slender features like network fields. In the Sun, the Wilson depression can amount to a few scale heights, and thus the measured fields in sunspots are $\sim 2 B_{\text {eq }}\left(T=T_{\text {eff }}\right)$, i.e., of order $3 \mathrm{kG}$ (although, sometimes, values as high as $5 \mathrm{kG}$ have been measured). In complete analogy with the Sun, one should not expect $B_{\text {eq }}\left(T=T_{\text {eff }}\right)$ to be a useful limit - within a factor of a few - to the fields in stellar dark spots. The equipartition argument still applies; however, to derive a useful limit on $B$ it is necessary to know the detailed thermal structure of the spot, a problem that still lacks a full solution even for the Sun.

\section{Comparison with Current Measurements}

At present, only two set of magnetic-field measurements for $\mathrm{T}$ Tauri stars are available ${ }^{3}$ : the measurements by Basri et al. (1992) and Guenther (1997). These observations measure the broadening of FeI lines in the wavelength range 5000-7000 $\AA$; at these wavelengths, a dark spot with temperature $T_{\text {eff }}-\Delta T$ that covers a fraction $f_{s}$ of the surface of a star with $T_{\text {eff }}$ contributes a fraction $f_{s} B_{\lambda}\left(T_{\text {eff }}-\Delta T\right) /(1-$ $\left.f_{s}\right) B_{\lambda}\left(T_{\text {eff }}\right)$ of the total emission, where $B_{\lambda}$ is the Planck function. Using the typical values $f_{s}=0.3$, $\Delta T=1000 \mathrm{~K}$, and $T_{\text {eff }}=4000 \mathrm{~K}$ (see, e.g., Bouvier et al. 1993), one finds that starspots contribute less than $7 \%$ to the light in the range 5000-7000 $\AA$. Therefore, current measurements are insensitive to the magnetic fields in the center of dark spots, where $B$ can be larger than $B_{\text {eq }}$ at $T=T_{\text {eff }}$.

Figure 1 is a comparison between the lower limits to $B$ derived from the observations (because $f \leq 1$ ) and $B_{\text {eq }}$ calculated from (6) as a function of $T_{\text {eff }}$ and different surface gravities. Also shown is the value of $B_{\text {eq }}$ for the Sun; comparison of this value with the one derived from (6) for a G2 V star gives a measure of

\footnotetext{
${ }^{3}$ Donati et al. (1997) detected circular polarization in V410 Tau and HDE 283572 (V987 Tau), but they did not derive a value of $B$ from their measurements. Guenther \& Emerson (1996) attempted to measure the fields in Tap 35 and V410 Tau to demonstrate the potential of infrared lines in Zeeman studies, but they obtained upper limits to $B$ that are consistent with the measurements of Basri et al. (1992).
} 
the typical errors (Table 1) in the constant- $g$ loci in Figure 1 for the spectral types currently accessible to Zeeman-broadening measurements (spectral type K7 or earlier). Note that these errors are much smaller than the observational error bars.

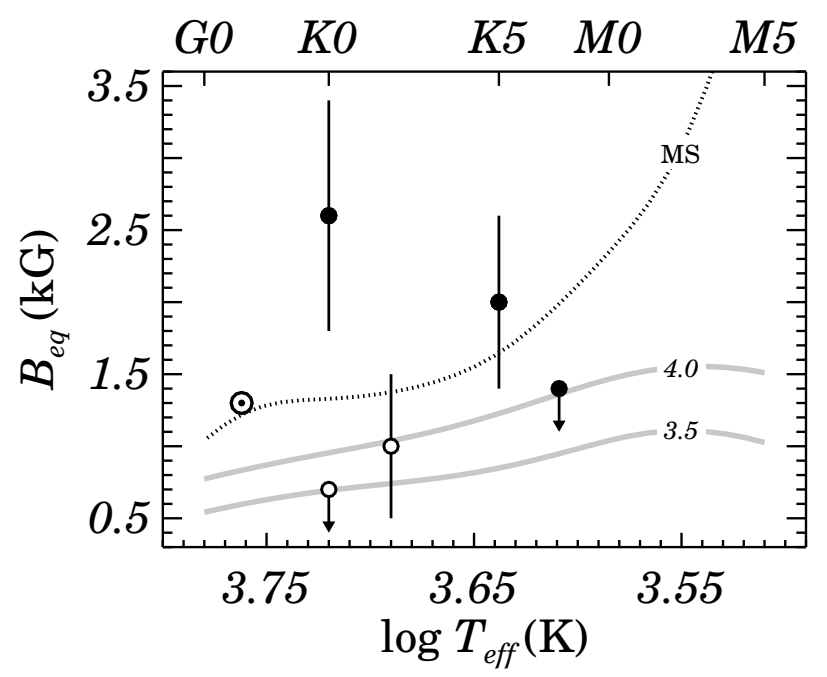

Fig. 1.- The equipartition magnetic field, $B_{\text {eq }}$, as a function of effective temperature $T_{\text {eff }}$ and different values of surface gravity $g$. Loci of constant $g$ are shown as grey lines and are labeled by the value of $\log g$. The field strengths derived by Guenther (1997) are shown as filled circles (from left to right): T Tau, Lk Ca 15, and Lk Ca 16; the values of $B$ from the observations by Basri et al. are shown as white circles (from left to right): TAP 10 and TAP 35. The arrows indicate upper limits. Also shown is the locus of $B_{\text {eq }}\left(T_{\text {eff }}\right)$ for the main-sequence (dotted line) and the value of $B_{\text {eq }}$ for the Sun from the Guenther et al. (1992) model is indicated by $\odot$.

There is a troubling trend in Figure 1, which is most obvious for the Guenther (1997) data. The fieldstrengths derived by this author are above the mainsequence values of $B_{\text {eq }}$. In other words, the only way for these objects to have magnetic fields as strong as implied by the data is for them to be more compact $(\log g \gtrsim 4.5)$ than a main-sequence star of the same spectral type. Obviously, this is impossible; moreover, $\log g \lesssim 3.5$ for classical TTS such as those in the Guenther (1997) dataset, and, also, the absorption lines form at $\tau<2 / 3$.
Furthermore, since I have assumed $f=1$ in deriving a value of $B$ from the measured $f B$, these values, again, are lower limits to the field implied by the observations. Therefore, the measurements of Guenther (1997) seem to overestimate $B$ by at least a factor of $>2\left(\frac{2 / 3}{\tau}\right)^{1 / 2}$ in the case of Lk Ca 15 and a factor $>2.5\left(\frac{2 / 3}{\tau}\right)^{1 / 2}$ for $\mathrm{T}$ Tau, respectively.

The measurements of Basri et al. (1992) for TAP 35 seem compatible with the expected $B_{\text {eq }}$, in particular because they assumed $\log g=4.0$ in their models. However, if $f<0.65$ their measurements would imply a value of $B$ larger than the equipartition field in a main-sequence star of the same spectral type. Moreover, they found that if $\log g=3.5$, instead of $\log g=4.0$, then a stronger field is required to match the data; this is inconsistent with the dependence of $B_{\text {eq }}$ on $g$ at fixed $T_{\text {eff }}$ (Figure 1). Therefore, there are also problems with the Basri et al. (1992) measurements, and the implied fields are larger than $B_{\text {eq }}$.

Another perspective on this inconsistency is presented in Figure 2. This figure is a plot of $B_{\text {eq }}$ as a function of $T_{\text {eff }}$ along the pre-main-squence isochrones of D'Antona \& Mazzitelli (1994); also shown are the paths in the $\left(T_{\text {eff }}, B_{\text {eq }}\right)$-plane traced by two stars with $M=1.5 \mathrm{M}_{\odot}$ and $M=0.4 \mathrm{M}_{\odot}$, respectively, as they evolve towards the zero-age main-sequence. These results show that, for a given value of the stellar mass, $B_{\text {eq }}$ increases with age; therefore, if one takes $\approx 10 \mathrm{Myr}$ as the age limit for the $\mathrm{T}$ Tauri phase, the results in Figure 2 imply that in TTS of spectral type $\mathrm{K} 7$ or earlier $B \lesssim 1.5 \mathrm{kG}$ outside dark spots.

\section{Conclusions}

The equipartition field $B_{\text {eq }}$ at any level in a stellar atmosphere is an upper limit to the magnetic field strength in a flux tube in pressure equilibrium with the surrounding, non-magnetized gas. I have computed $B_{\text {eq }}$ for the physical regime appropriate to $\mathrm{T}$ Tauri stars, and shown that the field strengths derived from current Zeemann-broadening measurements exceed this upper limit by large factors, and therefore are unphysical.

What are the causes for this discrepancy?

Because the derivation of $B$ from the measured line widths requires detailed modeling of the emergent line profiles, the fact that current models yield $B>B_{\text {eq }}$ indicates that some piece of physics is missing. To be fair to the Zeeman-broadening technique, when the 
Zeeman components are resolved this method can be very precise (see, e.g., the recent measurements of $B$ in $\epsilon$ Eri by Valenti et al., 1995), whereas Basri et al. (1992) based their analysis on the equivalent widths of the lines (due to the faintness of TTS and the high signal-to-noise required by this method), and Guenther (1997) used an autocorrelation analysis without benefit of a detailed atmospheric model. However, it is hard to imagine how the use of equivalent widths rather than line profiles can explain the finding by Basri et al. (1992) that a lower surface gravity requires a larger magnetic field to match the data, when the field is supposed to decrease with decreasing gravity (see eq. [6]). Therefore, the source of the discrepancy is, most likely, the input physics of the models.

In particular, current models for TTS use the same atmospheric structure for the magnetic and nonmagnetic parts of the photosphere, whereas at any given height the gas pressure in a flux tube has to be lower than that of its surroundings for pressure equilibrium to obtain. Because the measured field depends on the geometrical height where the lines are formed, and the flux tubes must be highly evacuated, using the same atmospheric structure for the magnetized and quiet regions of the photosphere is not correct, and is the most likely origin of the discrepancy. Therefore, this and other refinements (see Landolfi et al. 1989) must be incorporated to derive a more accurate estimate of $B$ in TTS. In addition, Valenti et al. (1995) showed that the Zeeman-broadening method is much more immune to the details of the model atmosphere when infrared lines are used. Infrared measurements should be pursued also, and one hopes that the discrepancy with the results presented here will disappear.

I wish to thank Robert Rosner for a conversation during which the idea for this paper was conceived, and Lee Mundy, Steve Stahler, and Stephen White for their careful reading of the manuscript. I am also indebted to an anonymous referee, who provided comments and criticisms that significantly improved an earlier version of this paper. This work was supported by NSF grant AST9613716 to the Laboratory for Millimeter-Wave Astronomy at the University of Maryland.

\section{REFERENCES}

Alexander, D. R. \& Ferguson, J. W. 1994, ApJ, 437, 879
Allard, F. \& Hauschildt, P. H. 1995, ApJ, 445, 433

Babcock, H. W. 1960, ApJ, 132, 521

Basri, G., Marcy, G. W., \& Valenti, J. A. 1992, ApJ, ,390, 622

Basri, G. \& Marcy, G. W. 1994, ApJ, 431, 844

Bouvier, J., Cabrit, S., Fernandez, M., Martin, E. L., \& Matthews, J. M. 1993, A\&A, 272, 176

Biermann, L. 1941, Vierteljahrsschr. Astron. Ges., 76,194

Cattaneo, F. \& Malagoli, A. 1992, in 7th Cambridge Workshop on Cool Stars, Stellar Systems, and the Sun, eds. M. S. Giampapa \& J. A. Bookbinder, ASP Conference Series Vol. 26, pg. 139

Cox, J. P. \& Giuli, R. T. 1968, Principles of Stellar Structure (New York: Gordon \& Breach), Vol. 2, pg. 590

D’Antona F. \& Mazzitelli I. 1994, ApJS, 90, 467

Donati J-F., Semel M., Carter B. D., Rees D. E., \& Collier Cameron A., 1997, MNRAS, 291, 658

Guenther, D. B., Demarque, P., Kim, Y.-C., \& Pinsonneault, M. H. 1992, ApJ, 387, 372

Guenther, E. W. 1997, in Herbig-Haro Flows and the Birth of Low Mass Stars, eds. B. Reipurth \& C. Bertout (Dordrecht: Kluwer), pg. 465

Guenther, E.W. \& Emerson, J.P. 1996, A\&A, 309, 777

Johnstone, R. M. \& Penston, M. V. 1986, MNRAS, 219, 927

Johnstone, R. M. \& Penston, M. V. 1987, MNRAS, 227,797

Küker M. \& Rüdiger G. 1997, A\&A, 328, 253

Landolfi M. et al. 1989, A\&A, 216, 113

Marcy, G. W. 1982, PASP, 94, 989

Robinson, R. D., 1980, ApJ, 239, 961

Parker, E. N. 1978, ApJ, 221, 368

Parker, E. N. 1979, Cosmical Magnetic Fields (Oxford:Clarendon)

Saar, S. H. 1988, ApJ, 324, 441

Saar, S. H. 1996, in IAU Symp. 176, Stellar Surface Structure, eds. K. G. Strassmeier \& J. L. Linsky (Dordrecht: Kluwer), pg. 237

Solanki, S. K. 1992, in 7th Cambridge Workshop on Cool Stars, Stellar Systems, and the Sun, eds. M. 
S. Giampapa \& J. A. Bookbinder, ASP Conference Series Vol. 26, pg. 211

Stein, R. F., Brandenburg, A., and Nordlund, A. 1992, in 7th Cambridge Workshop on Cool Stars, Stellar Systems, and the Sun, eds. M. S. Giampapa \& J. A. Bookbinder, ASP Conference Series Vol. 26, pg. 148

Valenti J. A., Marcy G. W., and Basri G. 1995, ApJ, 439,939
This 2-column preprint was prepared with the AAS LATEX macros $\mathrm{v} 4.0$.

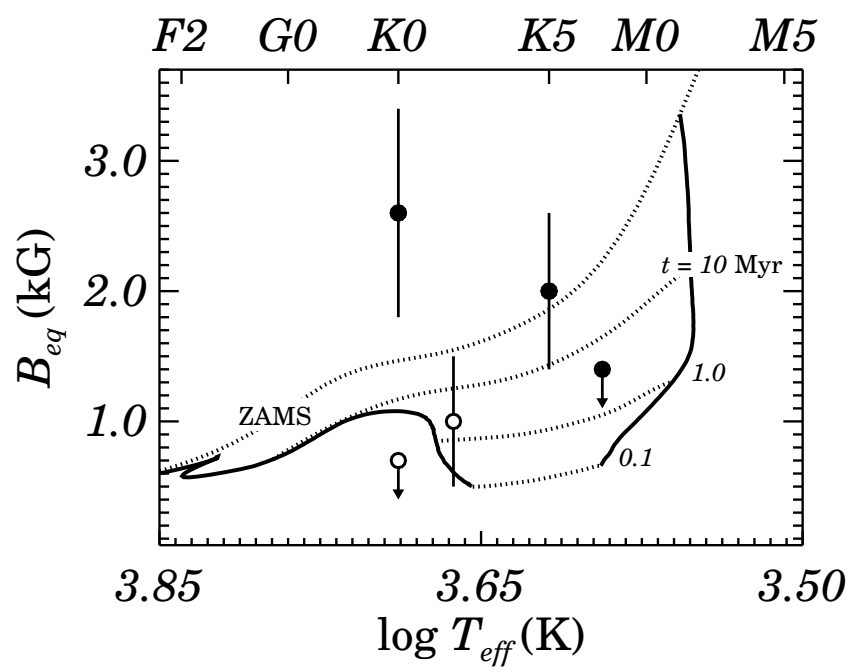

Fig. 2.- The equipartition magnetic field, $B_{\text {eq }}$, as a function of effective temperature $T_{\text {eff }}$ along the premain-sequence isochrones of D'Antona \& Mazzitelli (1994). The loci of constant age $t$ are shown as dotted lines and are labeled by the value of $t$. The solid lines indicate the paths in the $\left(T_{\text {eff }}, B_{\text {eq }}\right)$-plane for a $1.5 \mathrm{M}_{\odot}$ (left) and a $0.4 \mathrm{M}_{\odot}$ star (right) as they evolve towards the zero-age main-sequence. The other symbols have the same meaning as in Figure 1. 